The author also gratefully acknowledges the help of Professor W. R. Sears and Dr. Kuo in studying an earlier draft of this paper and in particular their advice regarding the section on the physical solutions.

\title{
REFERENCES
}

1. P. Molenbroek, Über einige Bewegungen eines Gases bei Annahme eines Geschwindigkeitspotentials, Archiv. Math. Phys. 9, 157-195 (1890).

2. S. A. Chaplygin, On gas jets, Ann. Imp. Univ. Mosc. (Phys-Math.) 21, 1-121 (1904).

3. H. S. Tsien, The limiting line in mixed subsonic and supersonic flow of compressible fluids, N.A.C.A. Tech. Note 961 (1944).

4. R. Courant and K. O. Friedrichs, Supersonic flow and shock waves, Interscience Publishers Inc., New York, 1948, p. 62.

5. J. Craggs, The breakdown of the hodograph transformation, Proc. Camb. Phil. Soc. 44, 360-379 (1948).

6. K. O. Friedrichs and D. Flanders, On the non-occurrence of a limiting line in transonic flow, Com. App. Math. (1) 3, 287-301 (1948).

7. A. R. Manwell, A method of variation for flow problems, Quart. Journ. Math. (Oxford) 20, 166-189 (1949).

8. F. I. Frankl, On the formation of shock waves in subsonic flows with local supersonic velocities, (Transl.), N.A.C.A. Tech. Mem. 1251.

9. G. Guderley, On the transition from a transonic potential flow to a flow with shocks, Air. Mat. Comm., T-2, F-TR-2160, ND.

\section{NOTE ON A SUFFICIENT CONDITION FOR THE STABILITY OF GENERAL, PLANE PARALLEL FLOWS*}

By MARTIN LESSEN (The Pennsylvania State College)

In reference 1, Synge derived sufficient conditions for the stability of plane Couette motion and plane Poiseuille motion. In the present note, it is demonstrated that similar conditions exist for any parallel flow with either finite or infinite boundary conditions or both. Although it can be shown that parallel two-dimensional motion must be of either Poiseuille or Couette type, it has been assumed, for purposes of stability considerations, that boundary-layer flows are substantially parallel in nature and Synge's treatment has therefore been extended to cover these flows.

The equation describing the stability of parallel flows for small disturbances is the Orr-Sommerfeld equation (reference 2 ):

$$
(w-c)\left(\varphi^{\prime \prime}-\alpha^{2} \varphi\right)-w^{\prime \prime} \varphi=-\frac{i}{\alpha R}\left(\varphi^{\mathrm{IV}}-2 \alpha^{2} \varphi^{\prime \prime}+\alpha^{4} \varphi\right),
$$

where $x$ is the coordinate in direction of the flow, $y$ the coordinate perpendicular to this direction, $t$ the time, $w=w(y)$ the velocity of steady flow, $\varphi(y) e^{i \alpha(x-c t)}$ the disturbance stream function; $c$ the disturbance phase velocity, $\alpha$ the disturbance wave number, $R$ the Reynolds number of steady flow.

${ }^{*}$ Received July $2,1951$. 
If the boundaries (finite or infinite) of the flow field are at $y_{1}$ and $y_{2}$, the boundary conditions on the disturbance amplitude function $\varphi$ are given by

$$
\begin{aligned}
\varphi\left(y_{1}\right) & =0, \quad \varphi\left(y_{2}\right)=0, \\
\varphi^{\prime}\left(y_{1}\right) & =0, \quad \varphi^{\prime}\left(y_{2}\right)=0 .
\end{aligned}
$$

If Eq. (1) is cleared of fractions, multiplied by $\bar{\varphi} d y$, integrated over the flow field, and the resulting equation added to its complex conjugate the following inequality corresponding to Eq. (11.16) of Ref. 1 is obtained.

$$
\alpha R c_{i}\left(I_{1}^{2}+\alpha^{2} I_{0}^{2}\right)<\alpha R q I_{0} I_{1}-\left(I_{2}^{2}+2 \alpha^{2} I_{1}^{2}+\alpha^{4} I_{0}^{2}\right),
$$

where

$$
\begin{array}{ll}
I_{0}^{2}=\int_{y_{1}}^{y_{2}} \varphi \bar{\varphi} d y, \quad I_{1}^{2}=\int_{y_{1}}^{y_{2}} \varphi^{\prime} \bar{\varphi}^{\prime} d y, \\
I_{2}^{2}=\int_{y_{1}}^{y_{2}} \varphi^{\prime \prime} \bar{\varphi}^{\prime \prime} d y, \quad q=w_{\max }^{\prime}, \quad c_{i}=\operatorname{Im} c .
\end{array}
$$

It should be noted that the values of all the integrals specified are convergent for infinite boundary conditions. This can be seen by considering the asymptotic properties of $\varphi$ at $y \rightarrow \pm \infty$ in the integrals.

$$
\varphi(y \rightarrow \pm \infty) \cong \text { constant } \times\left(e^{\mp \alpha \nu}\right)
$$

For a discussion of the asymptotic properties of $\varphi$, see reference (3).

The following inequality is now considered in place of (11.17) of Ref. 1 because the latter cannot be used for boundary conditions at infinity.

$$
\int_{y_{1}}^{y_{2}}\left|\varphi+\xi \varphi^{\prime}+\eta \varphi^{\prime \prime}\right|^{2} d y>0
$$

$\xi, \eta$ are real, arbitrary constants. It follows that

$$
\eta^{2} I_{2}^{2}>\left(2 \eta-\xi^{2}\right) I_{1}^{2}-I_{0}^{2} .
$$

If the inequality (3) is substituted into the inequality (2), the following relation results.

$$
\alpha R c_{i} \eta^{2}\left(I_{1}^{2}-\alpha^{2} I_{0}^{2}\right)<\alpha R q \eta^{2} I_{0} I_{1}-I_{1}^{2}\left(2 \eta-\xi^{2}+2 \alpha^{2} \eta^{2}\right)-I_{0}^{2}\left(\alpha^{4} \eta^{2}-1\right) .
$$

If $c_{i}>0$ then

$$
\left(\alpha R q \eta^{2}\right)^{2}>4\left(2 \eta-\xi^{2}+2 \alpha^{2} \eta^{2}\right)\left(\alpha^{4} \eta^{2}-1\right)
$$

where

$$
2 \eta-\xi^{2}+2 \alpha^{2} \eta^{2}>0, \quad \alpha^{4} \eta^{2}-1>0 .
$$

The sufficient condition derived for stability of general, plane parallel flows is a very weak one but it has the advantage of being extremely simple. The condition derived is 
weaker than the one obtained by Synge but it has wider application. Such two-dimensional, parallel flows as boundary layers, jets, and wakes can be quickly considered preliminary to more detailed investigation.

Consider Eq. (4) as applied to the laminar boundary layer between parallel streams (Ref. 3).

Let $\eta=K / \alpha^{2}, \xi^{2}=0$. Equation (6) then becomes

$$
(R q)^{2}>8\left(1+\frac{1}{K}-\frac{1}{K^{2}}-\frac{1}{K^{3}}\right) \alpha^{4}
$$

If the right hand side is maximized with respect to $K$, the corresponding value of $K$ is $K=3$. The value of $q$ for the problem under consideration is

$$
q=0.2000 \text {. }
$$

Therefore, for certain stability

$$
R<15.8 \alpha^{2} .
$$

It is interesting to note that, although no lower branch of the stability curve is obtained by the above method, the actual calculations to a second approximation for the free boundary layer also do not yield a lower branch of the neutral curve.

\section{REFERENCES}

1. J. L. Synge, Hydrodynamical stability. Vol. 2, Semicentennial Pub., Am. Math. Soc. (New York), 1938, pp. 227-269.

2. C. C. Lin, On the stability of two-dimensional parallel flows. Part I-general theory. Q. Appl. Math., 3, 117-142 (1945).

3. M. Lessen, On the stability of the free laminar boundary layer between parallel streams, NACA T. R. 979 .

\section{GENERALIZATION OF A PROBLEM OF RAYLEIGH*}

\section{By STANLEY CORRSIN (Department of Aeronautics, The Johns Hopkins University)}

Emmons has recently published a note [1] giving dissipative temperature distributions for Rayleigh's classic problem of the incompressible viscous flow set up by an infinite plate suddenly moved at constant velocity in its own plane [2]. This suggests sufficient interest to warrant exposition of a particular solution of the problem for a compressible fluid. This was worked out several years ago as a simple pedagogical demonstration of some characteristics of the compressible boundary layer, much as the Rayleigh case is often used to introduce students to the incompressible boundary layer.

We shall restrict the problem by taking low Mach number, $v / u \ll 1$, insulated wall, Prandtl number unity, perfect gas, $\mu \sim \lambda \sim T^{3 / 4}$ and $c_{p}=$ constant. The plate lies on the plane $y=0$ and moves in the $x$-direction; $u$ is velocity along $x, \nu$ is along $y ; c_{p}, \mu$, $\lambda, \rho, T$ are specific heat at constanit pressure, viscosity, thermal conductivity, density and absolute temperature, respectively.

${ }^{*}$ Received July 5, 1951. 